\title{
Changing Landscape of Cholangiocarcinoma
}

Ashish Saharia ${ }^{1 *}$, Constance Mobley ${ }^{1}$, Mark Hobeika ${ }^{1}$, Robert McMillan ${ }^{1}$, Asad Shaikh $^{1}$, Flavio Baio ${ }^{1}$, Maen Abdelrahim ${ }^{2}$, Kirk Heyne ${ }^{2}$, Mark Ghobrial ${ }^{1}$ and AO Gaber $^{1}$

${ }^{1}$ Department of Surgery, Houston Methodist Hospital, Houston TX, USA

${ }^{2}$ Department of Medical Oncology, Houston Methodist, Hospital, Houston TX, USA

ISSN: 2637-773X

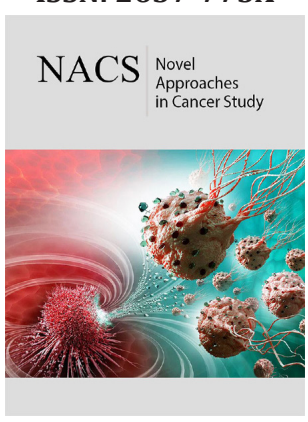

*Corresponding author: Ashish Saharia, Department of Surgery, Houston Methodist Hospital, Houston TX, USA

Submission: 沮 March 02, 2020

Published: 觜 March 06, 2020

Volume 4 - Issue 2

How to cite this article: Ashish Saharia, Constance Mobley, Mark Hobeika, Robert McMillan, Asad Shaikh, Flavio Baio, et al. Changing Landscape of Cholangiocarcinoma. Nov Appro in Can Study.4(2). NACS.000584.2020.

DOI: 10.31031/NACS.2020.04.000584

Copyright@ Ashish Saharia. This article is distributed under the terms of the Creative Commons Attribution 4.0 International License, which permits unrestricted use and redistribution provided that the original author and source are credited.

\begin{abstract}
Cholangiocarcinoma occur in cholangiocytes, either in the intrahepatic, peri-hilar or distal bile duct locations. The three, command distinct attention due to difference in presentation and management. The current 5 years survival for localized intrahepatic cholangiocarcinoma is estimated to be at $24 \%$. With the advent of molecular diagnostics and targeted therapy the field has seen some changes. Surgical resection is still the mainstay of therapy, although transplantation has been proposed in selected patients that have shown disease stability. Localized therapy for intra-hepatic and hilar tumors with neoadjuvant chemotherapy has also been used with some success for smaller tumors.
\end{abstract}

\section{Introduction}

The annual incidence of cholangiocarcinoma in the United States from 2001 to 2015 was 1.26 per 100,000 people per year. The incidence rates have increased over this time period, specifically for intrahepatic cholangiocarcinoma. According to the SEER data base, the average 5 -year survival rate of localized intra-hepatic cholangiocarcinoma is $24 \%$ and that of extrahepatic is $15 \%$. Native Americans have the highest incidence in the United States, about 6 times more than non-natives [1]. Occupational exposure to 1,2 DCP and or dichloromethane has been documented in people working in the printing industry in Japan [2]. Chlonorchis Sinensis, oriental liver fluke; Opisthorchis Viverrini as well as chronic hepatolithiasis increase chance of developing the disease [3].

Intrahepatic cholangiocarcinoma occurs above the second order bile ducts and has a median survival of 10 months. The only hope for cure is with surgical resection, although $2 / 3$ rds of the patient have disease recurrence in the remnant liver [4]. Advancements in molecular diagnostics and targeted therapy have mostly occurred in the field of intrahepatic cholangiocarcinoma [5]. Perihilar cholangiocarcinoma occurs below the second order bile ducts and above the level of cystic duct and poses the problems of difficulty in reaching diagnosis in advanced inoperable stages. It is frequently hard to obtain tissue from the ducts which in turn causes delay in treatment. Current treatment includes Gemcitabine based chemotherapy with localized therapy in the form of radiation or rarely surgery. Transplantation is offered for tumors less than $3 \mathrm{~cm}$, with negative nodes. Distal cholangiocarcinoma and gallbladder cancers are mostly unchanged, with pancreaticoduodenectomy and radical cholecystectomy being the most effective therapy in qualified clinical cases. Gemcitabine-based chemotherapy is most likely to benefit in the adjuvant setting.

This mini review will focus on evolving role of molecular diagnostics and transplantation.

\section{Molecular Diagnostics}

A new era of genetic profiling began with sequencing of human genome in 2003. Today, with the advent of Next Generation Sequencing, three billion base pairs can be analyzed in a matter of hours. Multiple tests can be performed in a limited amount of tissue. Millions of fragments of DNA are analyzed in parallel. In a matter of 2 weeks the genome can be tested for a comprehensive set of cancer related genes. Comprehensive genetic profiling (CGP)of the tumors is increasingly being used and currently considered the key driver in personalized cancer care. CGP is also available for somatic cells, which can identify inheritable genetic changes. There are four types of genetic alterations that occur in cancer cell transformation 
a) base substitution b) insertion and deletion of base pairs c) uncontrolled copying of base alterations and d) re-arrangements of bases. Older techniques such as, single marker molecular tests (ex. Running specific BRAF or V600e tests) use PCR amplicon technology and multigene hotspot test (identifies only base insertions and deletions and substitutions and misses MSI, copied alterations, gene rearrangements and mutational burden) are not as effective as CGP.

Tumoral Mutations and Targeted Treatment in Intrahepatic Cholangiocarcinoma

Daniela Sia et al. [6] looked at RNA and exosome sequencing analysis in 107 iCCA patients and found that $70 \%$ of patients harbor one actionable mutation (FGFR2 fusions, IDH1/2, ARAF, KRAS, BRAF and FGF19). Similar results were seen by Lowery et al. [7] of the 195 patients, 78\% intrahepatic tumors had mutations. The most common mutations found in iCCA were IDH1 (30\%), ARID1A (23\%), BAP1 (20\%), TP53 (20\%) and FGFR2 gene fusions (14\%) most of them were mutually exclusive. Rizvi et al. [8] found protein kinase cAMP- activated catalytic subunit alpha (PRKACA) or beta (PRKACB) gene fusion in pCCA and ELF3 mutations in dCCA/ ampullary cancer (Table 1 ).

Table 1: Mutations observed in iCCA, pCCA and dCCA their targeted therapies.

\begin{tabular}{|c|c|}
\hline Mutations & Targeted Therapy \\
\hline IDH1/2 & Ivosidinib \\
\hline FGFR1/2/3 & Erdafitinib \\
\hline BAP1 & Olaparib \\
\hline ARID1A/ARID1B & Tazemetostat \\
\hline BRAF & Vamurefenib \\
\hline KRAS & Selumetinib \\
\hline CDKN2A/CCND1 & CDK 4/6 inhibitors \\
\hline
\end{tabular}

A. IDH- Isocitrate dehydrogenase,

B. FGFR - Fibroblastic growth factor,

C. BAP- BRCA associated protein,

D. CDK- Cyclin dependent kinase,

E. CCND- Cyclin D.

IDH1 inhibitor, Ivosidinib is currently approved as a treatment for AML and appears to be effective against cholangiocarinoma with this mutation. In a multicenter phase 1 study between US and the Eastern cooperative oncology group, 73 patients were studied in a dose escalation trial (from 200-1200 mg per day). 500mg per day was used for the expansion and a median overall survival was found to be 13.4 months. Twelve-month progression free survival was $21.8 \%$ [9]. ARID1a mutations are associated with increased immune activity in GI cancers and is correlated with microsatellite instability. These cancers were also likely to express PD-L1 than
ARID1A- wild type GI cancers. This increases their chances to be susceptible to immune checkpoint blockade therapy [10].

BAP1 (BRCA1 associated protein 1)/ BRCAness - plays a role in repairing damaged DNA and controlling the activity of genes. BAP1 inhibition is demonstrated with PARP inhibitor Olaparib, especially when combined with cisplatin (studied in patients with mesothelioma) [10]. FGFR mutations: FGFR inihibitor, Infigratinib was used to treat cholangiocarcinoma but acquired resistance, which was found to be overcome with mTOR inhibitors, suggesting using combination Infigratinib -mTOR inhibitor therapy [11].

\section{Immunotherapy in Cholangiocarcinoma}

Expression of $\mathrm{B} 7 \mathrm{H} 1$ and $\mathrm{PD} 1$ was upregulated in the cholangiocarcinoma cells compared to the surrounding stroma. $\mathrm{B} 7 \mathrm{H} 1$ expression is related to immune evasion and its expression correlated with higher TNM stage and is inversely proportional to CD8 tumor infiltrating lymphocytes. Exploiting immunotherapy for the treatment of cholangiocarcinoma offers another avenue for therapy [12].

\section{Cell Free DNA in Cholangiocarcinoma}

Those managing cholangiocarcinoma realize that its diagnosis is particularly difficult. Intra-hepatic cholangiocarcinoma is usually read as undifferentiated adenocarcinoma on biopsy and perihilar cholangiocarcinoma is hard to biopsy, sometimes requiring multiple ERCP attempts. When spilled DNA can be picked from blood samples and the DNAs have special tumor signatures (in the form of mutations) a diagnosis can be supported, and treatment initiated. Potential application of cell free DNA is a) early diagnosis of tumor b) monitoring tumor heterogeneity c) identification of therapeutic targets d) real-time evaluation of tumor resistance or relapse [13]. In 2019, Mody et al. [14] reported the largest series profiling the ct DNA in patients with HCC and biliary tract tumor. Top few alterations found were TP53(18\%), TERT (14\%), CTNNB1 (13\%). Wasegang compared CfDNA methylation on patients with cholangiocarcinoma and other biliary diseases and found HOXD9 and OPCML as the mutation's diagnostic of cholangiocarcinoma [15].

\section{Transplantation for Cholangiocarcinoma}

Traditionally iCCA is considered a contraindication for liver transplantation, although small cholangiocarcinoma found incidentally in explanted livers were found to have good overall outcomes. UNOS gives exception points for perihilar cholangiocarcinoma for localized tumors less than $3 \mathrm{~cm}$. These patients get neoadjuvant chemotherapy, with and without radiation to keep the tumor under control until the time of transplant and treated post-transplant with adjuvant chemotherapy when indicated. Mayo protocol calls for 5FU based chemotherapy, external beam radiation with brachytherapy boosts in hilar cholangiocarcinoma [16]. Trans-peritoneal biopsies are contraindicated, because it is likely to cause tract implantation. Percutaneous biliary drainage is not without risk of local spread 
but is generally not observed.

Our group with MD Anderson treated intrahepatic cholangiocarcinoma patients with stable disease (irrespective of size and number) with liver transplantation. Overall survival at three years of these selected patients was found to be $83.3 \%$ with a $50 \%$ recurrence free survival at 1, 3 and 5 years. Patients with mutation were treated with targeted therapy and those with residual disease were given post-transplant chemotherapy. Extrahepatic recurrence was observed in those who retrospectively had lesions that were not considered significant [17].

\section{References}

1. Patel N, Benipal B (2019) Incidence of cholangiocarcinoma in the USA from 2001 to 2015: A US cancer statistics analysis of 50 states. Cureus 11(1): e3962.

2. Mimaki S, Totsuka Y, Suzuki Y, Nakai C, Goto M, et al. (2016) Hypermutation and unique mutational signatures of occupational cholangiocarcinoma in printing workers exposed to haloalkanes. Carcinogenesis 37(8): 817-826.

3. Kim TS, Pak JH, Kim JB, Bahk YY (2016) Clonorchis sinensis, an oriental liver fluke, as a human biological agent of cholangiocarcinoma: A brief review. BMB Rep 49(11): 590-597.

4. Spolverato G, Vitale A, Cucchetti A, Popescu I, Marques HP, et al. (2015) Can hepatic resection provide a long-term cure for patients with intrahepatic cholangiocarcinoma? Cancer 121(22): 3998-4006.

5. Chong DQ, Zhu AX (2016) The landscape of targeted therapies for cholangiocarcinoma: Current status and emerging targets. Oncotarget $7(29)$ : 46750-46767.

6. Sia D, Villanueva A, Friedman SL, Llovet JM (2017) Liver cancer cell of origin, molecular class, and effects on patient prognosis. Gastroenterology 152(4): 745-761.

7. Lowery MA, Ptashkin R, Jordan E, Berger MF, Zehir A, et al. (2018) Comprehensive molecular profiling of intrahepatic and extrahepatic cholangiocarcinomas: Potential targets for intervention. Clin Cancer Res
24(17): 4154-4161.

8. Rizvi S, Gores GJ (2017) Emerging molecular therapeutic targets for cholangiocarcinoma. J Hepatol 67(3): 632-644.

9. Lowery MA, Burris HA, Janku F, Shroff RT, Cleary JM, et al. (2019) Safety and activity of ivosidenib in patients with IDH1-mutant advanced cholangiocarcinoma: A phase 1 study. Lancet Gastroenterol Hepatol 4(9): 711-720.

10. Li L, Li M, Jiang Z, Wang X (2019) ARID1A mutations are associated with increased immune activity in gastrointestinal cancer. Cells 8(7) pii: E678.

11. Krook MA, Lenyo A, Wilberding M, Barker H, Dantuono M, et al. (2020) Efficacy of FGFR inhibitors and combination therapies for acquired resistance in FGFR2-fusion cholangiocarcinoma. Mol Cancer Ther.

12. Ye Y, Zhou L, Xie X, Jiang G, Xie H, et al. (2009) Interaction of B7-H1 on intrahepatic cholangiocarcinoma cells with PD-1 on tumor-infiltrating T cells as a mechanism of immune evasion. J Surg Oncol 100(6): 500-504.

13. Diaz LA, Bardelli A (2014) Liquid biopsies: Genotyping circulating tumor DNA. J Clin Oncol 32(6): 579-586.

14. Mody K, Kasi PM, Yang JD, Surapaneni PK, Ritter A, et al. (2019) Feasibility of circulating tumor DNA testing in hepatocellular carcinoma. J Gastrointest Oncol 10(4): 745-750.

15. Wasenang W, Chaiyarit P, Proungvitaya S, Limpaiboon T (2019) Serum cell-free DNA methylation of OPCML and HOXD9 as a biomarker that may aid in differential diagnosis between cholangiocarcinoma and other biliary diseases. Clin Epigenetics 11(1): 39.

16. Gores GJ, Darwish Murad S, Heimbach JK, Rosen CB (2013) Liver transplantation for perihilar cholangiocarcinoma. Dig Dis 31(1): 126129.

17. Lunsford KE, Javle M, Heyne K, Shroff RT, Abdel-Wahab R, et al. (2018) Liver transplantation for locally advanced intrahepatic cholangiocarcinoma treated with neoadjuvant therapy: A prospective case-series. Lancet Gastroenterol Hepatol 3(5): 337-348. 